\title{
How much internalization of nuclear risk through liability insurance?
}

\author{
Schneider, Yves ; Zweifel, Peter
}

\begin{abstract}
An important source of conflict surrounding nuclear energy is that with a very small probability, a large-scale nuclear accident may occur. One way to internalize the associated financial risks is through mandating nuclear operators to have liability insurance. This paper presents estimates of consumers' willingness to pay for increased financial security provided by an extension of coverage, based on the "stated choice" approach. A Swiss citizen with median characteristics may be willing to pay 0.14 US cents per kwh to increase coverage beyond the current CHF 0.7 billion (bn.) (USD 0.47 bn.). Marginal willingness to pay declines with higher coverage but exceeds marginal cost at least up to CHF 4 bn. (USD 2.7 bn.). An extension of nuclear liability insurance coverage therefore may be efficiency-enhancing.
\end{abstract}

DOI: https://doi.org/10.1023/B:RISK.0000046144.89048.9e

Posted at the Zurich Open Repository and Archive, University of Zurich

ZORA URL: https://doi.org/10.5167/uzh-1213

Journal Article

Published Version

Originally published at:

Schneider, Yves; Zweifel, Peter (2004). How much internalization of nuclear risk through liability insurance? Journal of Risk and Uncertainty, 29(3):219-240.

DOI: https://doi.org/10.1023/B:RISK.0000046144.89048.9e 


\title{
How Much Internalization of Nuclear Risk Through Liability Insurance?
}

\author{
YVES SCHNEIDER \\ yschneider@soi.unizh.ch \\ PETER ZWEIFEL* \\ pzweifel@ soi.unizh.ch \\ Socioeconomic Institute, University of Zurich, Hottingerstrasse 10, CH-8032 Zurich, Switzerland
}

\begin{abstract}
An important source of conflict surrounding nuclear energy is that with a very small probability, a large-scale nuclear accident may occur. One way to internalize the associated financial risks is through mandating nuclear operators to have liability insurance. This paper presents estimates of consumers' willingness to pay for increased financial security provided by an extension of coverage, based on the 'stated choice' approach. A Swiss citizen with median characteristics may be willing to pay $0.14 \mathrm{US}$ cents per kwh to increase coverage beyond the current $\mathrm{CHF}$ 0.7 billion (bn.) (US\$ 0.47 bn.). Marginal willingness to pay declines with higher coverage but exceeds marginal cost at least up to CHF 4 bn. (US\$ $2.7 \mathrm{bn}$.). An extension of nuclear liability insurance coverage therefore may be efficiency-enhancing.
\end{abstract}

Keywords: risk, nuclear energy, liability insurance, internalization

JEL Classification: D62, D81, Q48

Nuclear power plants provoke conflicts in many countries. While many voters and politicians are committed on this issue, others will gauge the advantages and disadvantages of the nuclear option. On the downside, an important consideration is that with a very small probability, an accident causing billions of Dollars of damage may occur.

This paper seeks to estimate the willingness-to-pay (WTP) of Swiss citizens for relief from the financial consequences of a severe nuclear accident, to be provided by an extension of liability insurance coverage mandated to nuclear power operators. This statement of objective calls for two clarifications. First, the risk to be considered needs to be defined. In the production process of nuclear energy, at least ten stages can be identified, each with its proper risks (Hirschberg, Spiekerman, and Dones, 1998). This paper deals exclusively with the risks of nuclear energy produced in Switzerland. Second, it focuses on mandatory liability insurance as an instrument for risk internalization. The many norms that govern the production of nuclear power, monitored by the Swiss Nuclear Safety Inspectorate (HSK), are simply taken as given.

This regulation in combination with the legal norm of liability still leaves room for what Shavell (1986) calls the judgement proof problem. The judgement proof problem consists of the possibility that nuclear power plant operators may fail to pay compensation for the damage caused, due to lack of assets. As shown by Shavell, mandatory liability insurance 
serves to avoid this shortcoming. This finding justifies considering mandatory liability insurance as an instrument of nuclear risk internalization.

In Switzerland, the current coverage of CHF 0.7 billion (bn.) (approx. US $\$ 0.47$ bn. at 2002 exchange rates) written by private insurers will hardly be sufficient to compensate the victims of an accident. However, an extension of coverage will result in a higher outlay for nuclear power. No market for individually contracted supplementary coverage has developed, quite likely because there are no contractual relationships between nuclear insurers and consumers to build upon. Therefore, determining the importance accorded to more comprehensive relief from the financial consequences of a severe nuclear accident amounts to estimating the increase in the price of electricity that would be accepted by consumers in return for extended liability coverage to be bought by plant operators.

'Stated choice' rather than conventional 'contingent valuation' experiments were conducted to measure WTP. In 'stated choice', different attributes of electric power are distinguished and varied from one scenario to the next. This should avoid fixation on the price attribute which in 'contingent valuation' may cause respondents to forget about alternate uses of their income, thus resulting in excessive estimates of WTP. Moreover, respondents are not asked to provide rankings but only to choose between the status quo and one alternative at a time, which serves to bring the experimental situation close to everyday decision making.

The basic hypothesis is that opting for the alternative must entail a utility gain that can be related to the levels of the attributes pertaining to the scenario. Therefore, the utility associated with an attribute can be inferred from the observed choice sequences using a discrete choice model. Since the surcharge per kwh is one of the attributes, it is possible to calculate WTP values for the other attributes (in particular, extension of insurance coverage).

Indeed, respondents valued five attributes of power in a way that is consistent with economic theory, and median estimated WTP amounts to a realistic 0.14 US cents per kwh. Since quite probably the extra cost of a fivefold extension of present liability coverage is below this figure, the benefits of such an extension exceed its cost, suggesting an improvement of efficiency.

\section{Determining willingness to pay through 'stated choice'}

For goods and services traded on markets, there is no need to measure willingness to pay. By accepting a price the customer reveals that his WTP is at least as high as the price. The safety of nuclear power plants is not yet traded on markets (though it is possible to think of nukebonds which mature in case of a prespecified accident). Neither does individually contracted insurance against nuclear risks exist which would reveal potential victims' willingness to pay for nuclear safety.

\section{1. 'Stated choice' methodology}

In the absence of opportunities to observe revealed preferences, it is necessary to measure stated preferences through experiments. Earlier attempts to measure WTP for nonmarket 
goods used the contingent valuation variant of stated preference (see e.g. Mitchell, 1989; Hausman, 1993). This methodology can also be applied to risk reduction (see e.g. Krupnick et al., 2002).

In the present context, respondents would have to state the maximum amount per kwh they would be prepared to pay for the increased financial security achieved by an extension of mandatory liability insurance. The difficulty with this direct approach is that in real life people hardly ever ask themselves such questions. Rather, they compare the attributes of a good and its price and then decide to buy it or not.

The 'stated choice' approach, developed by Louviere and Hensher (1982), seeks to simulate this everyday decision making. Rather than directly asking for willingness to pay, it seeks to elicit it indirectly through 'accept'/'reject' choices. Its basic assumption is that individuals derive utility from the attributes of products and are willing to trade them off against each other (Lancaster, 1966). The first application of stated choice to risk reduction seems to be Telser and Zweifel (2002), which extends the paired comparison approach used in Magat, Viscusi, and Huber (1988) to a framework of multiple product attributes and ordinal utility.

In the present context, the 'stated choice' method allows individuals to choose among different types of electricity. During the decision process, the attributes (among them price) of electricity are traded off against each other. By observing several similar decisions it is possible to estimate how much income (through higher electricity prices) respondents are ready to give up in return for an increased amount of some other desired attribute.

One particular tradeoff is illustrated in Figure 1. Utility increases with insurance coverage in the event of an accident (as a percentage of maximum possible loss) and decreases with

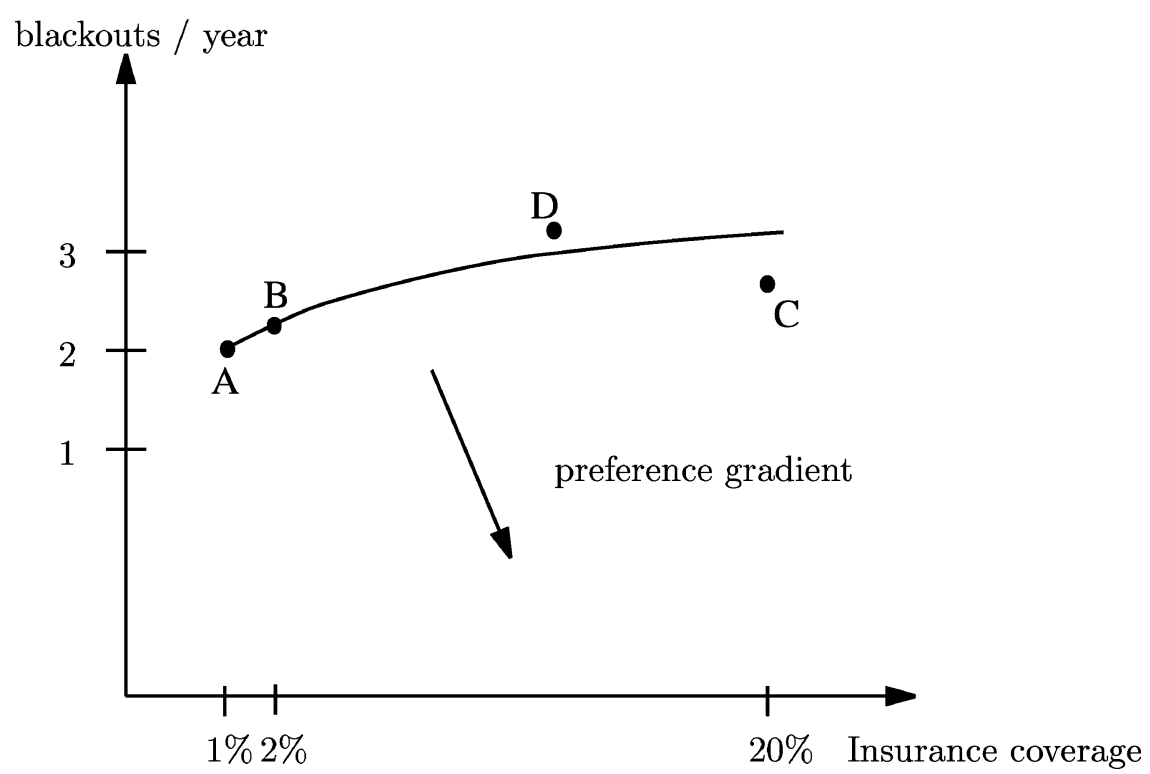

Figure 1. Trading off different product attributes. 
the average number of power blackouts; both attributes were found to be relevant to the persons interviewed (see Section 2). Assume that the status quo is given by combination A (1\% insurance coverage, 2 blackouts/year). Point B indicates that the individual is willing to accept a slightly higher number of blackouts if insurance coverage is raised to $2 \%$. The corresponding sacrifice in terms of security of supply is the marginal WTP for an increased insurance coverage.

Now participants in the experiment are asked to evaluate additional combinations, for example point $\mathrm{C}$. If $\mathrm{C}$ is accepted, then the individual's indifference curve must lie above C. Next, it must lie below point D if D is rejected. Proceeding in this manner, it is possible to approximate the indifference curve. Finally, the marginal willingness to pay measured in money terms (MWP) can be estimated in the same way, by introducing the increase in the price of electricity as an additional product attribute. The corresponding increase in the outlay on electricity is a sacrifice of income which would otherwise be available for spending on other goods.

Summing up, using 'stated choice' the experimenter is not limited to varying only price and insurance coverage but can introduce other attributes that influence real life decisions concerning electricity. Failing this, he runs the risk of causing respondents to associate with variations in price or insurance coverage attributes not explicitly included in the experiment (and therefore assumed as fixed).

\subsection{Problems with risk assessment}

The ultimate objective of this work is to provide guidance to public regulation. For this purpose it would be preferable to have experiments based on objective probabilities. However, in the present context objective probabilities are not available since experts differ in their estimates of the probability of a catastrophic nuclear accident (Zweifel and Umbricht, 2002). In this situation, respondents' own subjective estimates become crucially important. Specifically the additional ambiguity caused may affect respondents' level of expected utility as well as their tradeoffs between attributes such as the one shown in Figure 1 (Viscusi, 1998, ch. 2).

Ganderton et al. (2000) provide a possible solution to the problem of ambiguity. They conduct laboratory experiments where subjects had to decide about insurance against a low probability-high consequence event. Several draws from a loss function known to participants were revealed to subjects, who made choices largely consistent with predictions of the expected utility theory. However, the loss function cannot be claimed to be known in the present context.

Another problem is low probabilities. Here, Kunreuther, Novemsky, and Kahneman (2001) found that supplying a reference point in terms of a more probable and familiar risk and providing a good deal of contextual information helped respondents in dealing with low probabilities. In the present study, experts' average estimated probability of the highest possible loss served as the reference point. However, respondents were not asked to adopt this value, but to indicate their own probability estimate relative to that of the experts. The required contextual information comes from two sources, viz. the introduction to the experiment (see Appendix 5.1) and the remaining attributes characterizing types of 
Frequency

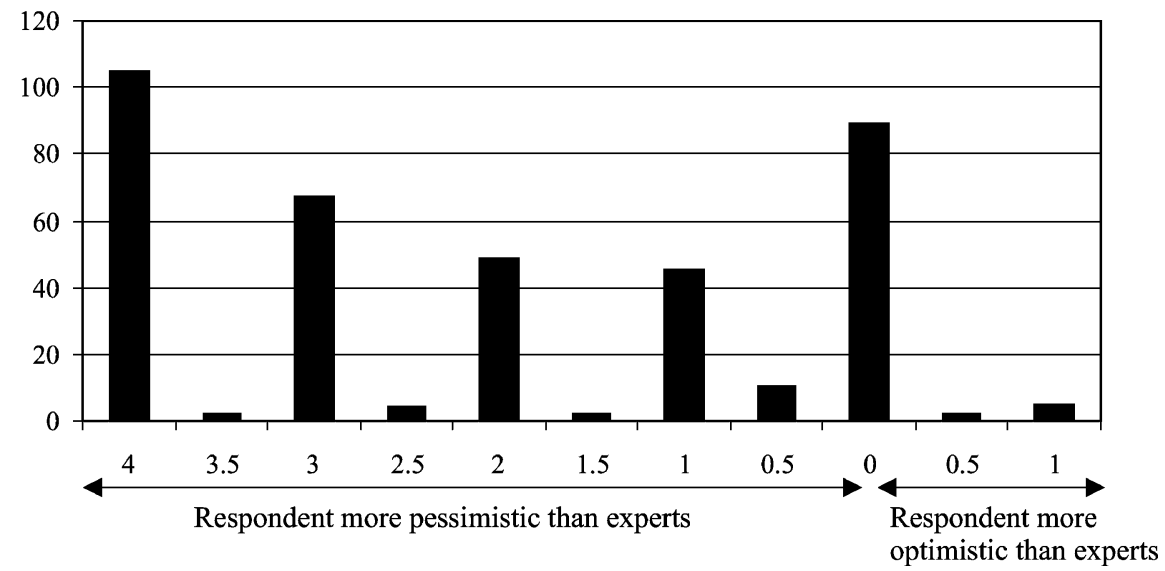

Figure 2. Beliefs about loss probabilities relative to experts' estimates. $X$-axis shows probability deviations in powers of 10 (see Appendix 5.2).

power, which moreover are allowed to vary during the 'stated choice' experiment. In sum, this setup serves to reduce the complexity of the choice situations by only incorporating the loss dimension of risk, while still allowing the effect of different beliefs about the loss probabilities to be estimated.

Figure 2 shows the frequencies of deviations from the reference point (the scale used to elicit this information can be found in Appendix 5.2). More pessimistic beliefs clearly outweigh optimistic deviations, in keeping with findings reported by Camerer and Kunreuther (1989).

\section{Experimental design}

In the context of a 'stated choice' experiment several issues must be addressed. What product attributes are to be included? How many levels should be distinguished in an attribute? How should the level of attributes vary between choice scenarios? How many choice scenarios should be presented to each respondent? An extensive literature is devoted to these and other issues (see e.g. Louviere, Hensher, and Swait (2000) and Hedayat, Sloane, and Stufken (1999)).

\subsection{Determination of attributes and their levels}

First of all, the relevant attributes of electric power need to be identified. Their number must be kept low for the decision problem to remain manageable. In a separate survey, approximately 500 persons were asked in spring 2001 to rate 15 different attributes, assigning them ranks between 1 (not important) and 10 (very important). Among the most important were: secure and sustainable waste disposal (9.26), size of area exposed to hazard (8.91), 
Table 1. Levels of attributes.

\begin{tabular}{llll}
\hline Attribute & \multicolumn{1}{c}{ Levels $\left(\mathrm{Coding}^{\mathrm{c}}\right)$} & \multicolumn{1}{c}{ Unit } & Status quo \\
\hline Price & $0 ; 10 ; 30 ; 60(\mathbf{0} ; \ldots ; 60)$ & percent & 0 \\
Blackouts & $2 ; 14(\mathbf{0} ; 1)$ & numb./year & 2 \\
Waste & unresolved problems $(\mathbf{1}) ;$ & & unresolved \\
& no unresolved problems $(0)$ & & problems \\
Damage $^{\mathrm{a}}$ & $0.1 ; 10 ; 100 ; 200(0.1 ; \ldots ; \mathbf{2 0 0})$ & CHF bn. & 200 \\
Coverage $^{\mathrm{b}}$ & $1 ; 20 ; 50 ; 100(\mathbf{1} ; \ldots ; 100)$ & percent & 1 \\
\hline
\end{tabular}

${ }^{a}$ Values in US\$ bn: $0.065 ; 6.5 ; 65 ; 130$ (at 2002 exchange rates).

${ }^{\mathrm{b}}$ Coverage in percent of loss.

${ }^{\mathrm{c} B o l d}$ for status quo.

reliability (low frequency of blackouts) (8.68), financial compensation of the victims in case of an accident (8.78), and average price per kwh (7.69).

Next, levels have to be assigned to attributes. Again they must be few in number in order to avoid long interviews. But then, the levels must reach sufficiently extreme values to cause respondents to switch from "accept" to "reject" and vice versa.

To test the questionnaire, six persons were interviewed in a first pretest. Without exception they understood the questions and were able to process the choice scenarios without problems. However, the attribute 'insurance coverage' was regarded as relatively unimportant.

A second pretest comprising 20 persons was conducted, with the maximum price hike boosted to 60 percent in order to induce a sufficient frequency of rejected scenarios (compared to the status quo). The most important attribute was again safe waste disposal, this time followed by insurance coverage and price.

For the final survey the five product attributes 'price', 'blackout', 'waste disposal', 'damage' and 'insurance coverage' were used. Table 1 gives an overview and the next section describes the attributes in detail.

\subsection{Description of attributes}

Price. The attribute 'Price' is the percentage increase caused by the extension of liability insurance coverage over the status quo. It read, e.g., "30 percent more expensive than at present (this is equivalent to a surcharge of CHF 285 on an annual electricity bill of CHF 950)". To obtain an absolute value for willingness to pay (WTP), this value was later multiplied by the actual annual electricity bill as indicated by respondents. Throughout the analysis it was assumed that respondents do not change the quantity of energy consumed. With this assumption, the higher price can be translated into a higher total outlay for electricity, and hence a lower disposable income which thus becomes a product attribute in the econometric analysis (see Section 3.2).

Blackout. The attribute 'Blackout' indicates if the scenario considered has a high incidence of blackouts (14 per year, coded 1 ) or a low incidence ( 2 per year, coded 0 ). It is a proxy 
of service reliability. The scenario description read, “... In Switzerland, blackouts are rare and mainly caused by environmental effects (storms) or maintenance work..."

Waste. Since 'Waste' was an important attribute in both pretests, it had to be included in the final experiment. This variable takes on two values: Either there are unresolved problems with waste disposal $(=1)$, or there are no unresolved problems with waste disposal $(=0)$. 'Waste' was described as follows, “... Disposal of waste occasions problems and risks of variable magnitude. This holds in particular for nuclear waste, where these problems are not resolved yet."

Damage. This indicates that electricity generation may cause a (hypothetical) maximum loss amounting to e.g. CHF $100 \mathrm{bn}$. (appr. US\$ 65 bn.) in the event of an accident. In order to make this amount more comprehensible, it was also expressed as an average damage per household. The attribute description said, “... All types of generating facility can cause accidents. Large scale accidents are rare. The magnitude of an accident cannot be calculated with precision but strongly depends on the type of facility."

Coverage. This indicates the part of maximum loss which would be covered by liability insurance. The text said, e.g., "one percent of the financial damage is insured". See Figure 3 for an example.

Note that the probability of an accident is not among the product attributes, as explained in Section 1.2.

Figure 3 shows one of the choice scenarios. Type A power is always associated with the status quo scenario to simplify decision making. Type B power has the same five attributes as type A, but with levels changed with regard to four of them. Thus, by simply deciding between types A and B, respondents implicitly trade off attributes. Since this choice is repeated several times with varying attribute levels for type $\mathrm{B}$, these tradeoffs become estimable.

\subsection{Scenario selection}

The design summarized in Table 1 gives rise to 256 possible scenarios. ${ }^{1}$ Obviously this is an excessive number for the questionnaire, calling for a reduced design. This was constructed using 'Gosset', a general purpose program for designing experiments (Hardin and Sloane, 1993) (see Appendix 5.3 for the program code). 'Gosset' selects the vectors of a regressor matrix $X$ in a way as to minimize or maximize a function of the covariance matrix of the parameters to be estimated, viz. $\left(X^{\prime} X\right)^{-1}$ in the case of OLS. The $D$-optimality criterion which was used in the present experiment, maximizes the determinant of the Fisher information matrix. While 'Gosset' solves this maximization problem for linear models (such as OLS) only, it is a reasonable approximation for nonlinear models such as probit (Kanninen, 2002). The reduced design was chosen to enable estimation of all quadratic as well as all interaction terms between product attributes. In this way, the number of scenarios to be included in the survey was reduced to 42 . 


\begin{tabular}{|c|c|c|}
\hline \multicolumn{3}{|c|}{ Decision No. 4209} \\
\hline & Type A power & Type B power \\
\hline Price & $\begin{array}{l}\text { A kilowatt hour costs the same } \\
\text { as today }\end{array}$ & $\begin{array}{l}\text { A kilowatt hour is } 60 \text { percent } \\
\text { more expensive than today }\end{array}$ \\
\hline Blackouts & $\begin{array}{l}2 \text { blackouts per } \\
\text { year on average }\end{array}$ & $\begin{array}{l}2 \text { blackouts per } \\
\text { year on average }\end{array}$ \\
\hline Waste & $\begin{array}{l}\text { There are unresolved problems } \\
\text { with waste disposal }\end{array}$ & $\begin{array}{l}\text { There are no unresolved } \\
\text { problems with waste disposal }\end{array}$ \\
\hline Damage & $\begin{array}{l}\text { A large scale accident can } \\
\text { cause losses up to a maximum } \\
\text { of Swiss francs } 200 \text { bn. (This } \\
\text { amounts to Swiss francs } 70,000 \\
\text { per household on average) }\end{array}$ & $\begin{array}{l}\text { A large scale accident can } \\
\text { cause losses up to a maximum } \\
\text { of Swiss francs } 100 \mathrm{mn} \text {. (This } \\
\text { amounts to Swiss francs } 35 \text { per } \\
\text { household on average) }\end{array}$ \\
\hline $\begin{array}{l}\text { Insurance } \\
\text { Coverage }\end{array}$ & $\begin{array}{l}1 \text { percent of this maximum } \\
\text { damage is covered }\end{array}$ & $\begin{array}{l}100 \text { percent of this maximum } \\
\text { damage is covered }\end{array}$ \\
\hline \multirow{2}{*}{ Your Choice } & Type A & Type B \\
\hline & \multicolumn{2}{|c|}{ cannot decide } \\
\hline
\end{tabular}

Figure 3. Example of a choice scenario.

Since it is not possible for a respondent to evaluate all 42 scenarios, 14 scenarios were chosen at random. This resulted in a unique 'stated choice' questionnaire for each person interviewed.

\subsection{Design of the questionnaire}

The questionnaire was divided into four parts. The first part consisted of some general warm-up questions concerning energy use. In addition the yearly electricity outlay of the respondent was asked for.

The second part contained information about the consequences of severe accidents in power production, focusing on nuclear and hydro (see Appendix 5.1 for details). In Switzerland fossil fuels are negligible in electricity generation. In addition, the limited coverage of current nuclear liability insurance was evoked. Two solutions to this problem were sketched. First, the government could raise tax to compensate victims in the event of an accident. Second, mandated insurance coverage could be extended, which however would result in higher electricity prices. For more details (in German), see http://www.soi.unizh.ch/ staff/schneider/index $2 . h t m l$. Respondents were asked to state their subjective probability of 
a severe accident relative to that of experts (see Appendix 5.2). Finally the attributes used in the stated choice part of the questionnaire were explained (see Section 2.2 above).

The third part contained the scenarios for the 'stated choice' experiment.

In the fourth part, socioeconomic data and information concerning the understanding of the 'stated choice' experiment were collected. Some 20 percent of respondents reported difficulties with the questionnaire. Roughly 73 percent stated they considered one of the attributes to be of overriding importance, which could be interpreted as an indication of lexicographic preferences. However, the econometric analysis failed to produce evidence suggesting that these individuals traded off attributes less frequently or less consistently.

\section{Econometric analysis}

\subsection{Data}

Face to face interviews were performed in the German speaking part of Switzerland during September and October 2001. In total, 391 persons were interviewed. Average income of respondents is CHF 42,000 (US\$ 28,000) having a yearly outlay on electricity of CHF 940 (US\$ 630). 80 percent had a medium level of education (either vocational school, community college, technical college or equivalent education) and the average age was 42 . Moreover, the sample was designed to have an equal proportion of men and women.

With 391 persons each evaluating 14 (out of a total of 42) choice scenarios, a total of 5,474 decisions were recorded. Respondents who felt unable to decide could always choose the option "not able to decide". This served to prevent choices made at random by individuals who in fact were indifferent or unable to decide.

In 819 cases (15 percent), no choice was stated, resulting in 4,655 usable observations. Only 90 percent of these $(4,154)$ were used for estimation, while 10 percent were put aside for an out-of-sample test (Section 3.5). In 27 percent of choices, the status quo was preferred. Missing values for socioeconomic information resulted in a final reduction to 4,119 observations.

\subsection{Theoretical background and specification}

No attempt was made to anchor specification in expected utility theory. Instead a general utility function in the retained attributes with linear, quadratic and mixed terms is used. To allow for heterogeneous preferences, socioeconomic characteristics of the respondents are interacted with income net of electricity outlay (i.e. disposable income), in accordance with Johnson and Desvousges (1997). This leads to the following utility function for individual $i$ and power type $j$ :

$$
U_{i j}=\alpha X_{j}+\beta_{1} w_{i j}+\beta_{2} w_{i j}^{2}+\gamma_{1} z_{i} w_{i j}+\gamma_{2} z_{i} w_{i j}^{2},
$$

where $X_{j}$ includes all linear, quadratic and mixed terms of electricity attributes, except disposable income $w_{i j}$. Disposable income is given by $w_{i j}:=\left(m_{i}-\right.$ outlay $\left._{i j}\right)$ with $m_{i}$ for 
income and outlay $_{i j}$ for total outlay on electricity, $z_{i}$ denotes the vector of socioeconomic variables, and $\alpha, \beta_{1}, \beta_{2}, \gamma_{1}, \gamma_{2}$ are to be estimated.

According to the random utility model (McFadden, 2001), the respondent evaluates the utility of the two scenarios and chooses the one with the higher utility. If ' $A$ ' denotes the attribute values of the status quo scenario, then individual $i$ chooses the alternative (B) of choice set $j$ if $U_{i B_{j}}>U_{i A_{j}}$, i.e. if

$$
\begin{aligned}
& \alpha \\
& \quad\left(X_{B_{j}}-X_{A_{j}}\right)+\beta_{1}\left(1-d_{i}\right)\left(w_{i B_{j}}-w_{i A_{j}}\right)+\beta_{2}\left(1-d_{i}\right)\left(w_{i B_{j}}^{2}-w_{i A_{j}}^{2}\right) \\
& \quad+\gamma_{1} z_{i}\left(1-d_{i}\right)\left(w_{i B_{j}}-w_{i A_{j}}\right)+\gamma_{2}\left(1-d_{i}\right) z_{i}\left(w_{i B_{j}}^{2}-w_{i A_{j}}^{2}\right) \\
& \quad+\delta_{1} d_{i}\left(w_{i B_{j}}-w_{i A_{j}}\right)+\delta_{2} z_{i} d_{i}\left(w_{i B_{j}}-w_{i A_{j}}\right) \\
& \quad+\delta_{3} d_{i}\left(w_{i B_{j}}^{2}-w_{i A_{j}}^{2}\right)+\delta_{4} d_{i} z_{i}\left(w_{i B_{j}}^{2}-w_{i A_{j}}^{2}\right)+\eta_{i}+\epsilon_{i j}>0 .
\end{aligned}
$$

In addition to Eq. (1), Eq. (2) contains a dummy variable $d_{i}$ to reflect the fact that income is missing with 43 percent of all individuals. It takes on the value of zero if income was revealed and one if missing, in which case the difference in disposable income reduces to the (inverse) difference of electricity outlay.

The error term appearing in this comparision has an individual-specific $\left(\eta_{i}\right)$ and a general component $\left(\epsilon_{i j}\right)$ that also varies with the choice set presented. The two components are assumed to conform to the usual random effects specification (Greene, 1997, ch. 14), with $\rho=\operatorname{var}\left(\eta_{i}\right) / \operatorname{var}\left(\eta_{i}+\epsilon_{i j}\right)$.

The dependent variable $y_{i j}$ (choice of power type B) is given by

$$
y_{i j}= \begin{cases}1 & \text { if inequality (2) holds } \\ 0 & \text { otherwise }\end{cases}
$$

From this expression it becomes clear that the variables used in estimation are the differences between the attribute levels of scenario B and scenario A. For instance damage is defined as the maximum level of loss in scenario B minus the maximum level of loss in scenario A. For example, an individual opting for type B power in decision no. 4209 of Figure 3 and having revealed his or her income has the following observation vector, in keeping with Table 1: ( $y$; blackout $;$ waste ; damage $;$ coverage $;. . . ;$ blackout $^{2} ;$ waste $^{2} ;$ damage $^{2} ;$ coverage $\left.^{2} ; . ..\right)$ $=\left(1 ; 0-0 ; 0-1 ; 0.1-200 ; 100-1 ; \ldots ; 0^{2}-0^{2} ; 0^{2}-1^{2} ; 0.1^{2}-200^{2} ; 100^{2}-1^{2} ; \ldots\right)$. Personal characteristics, which do not change between scenarios, drop out of the regression unless they interact with electricity attributes.

\subsection{Explanatory variables}

Table 2 shows descriptive statistics for some of the variables used. The product attributes were already explained in Section 2 . Note that outlay on electricity rather than price was used as an explanatory variable. In this way, an increase in outlay (occasioned by a higher price) can be interpreted as a reduction of disposable income. Of course, this holds only if outlay 
Table 2. Descriptive statistics for variables used in estimation.

\begin{tabular}{|c|c|c|c|}
\hline & Mean & Median & Unit \\
\hline blackouts $^{\mathrm{a}}$ & 0.48 & 0 & Dummy \\
\hline waste $^{\mathrm{a}}$ & -0.49 & 0 & Dummy \\
\hline damage $^{\mathrm{a}}$ & -128 & -190 & CHF bn. \\
\hline Coverage $^{a}$ & 43 & 49 & percent \\
\hline Disposable income ${ }^{\mathrm{a}}$ & -152 & 0 & $\mathrm{CHF}$ \\
\hline Damage $^{2 b}$ & $-28,175$ & $-39,900$ & CHF bn. \\
\hline Coverage $^{2 b}$ & 3,360 & 2,500 & percent \\
\hline Outlay on electricity & 940 & 840 & $\mathrm{CHF}$ \\
\hline Income (if revealed) & 41,890 & 36,000 & $\mathrm{CHF} /$ year \\
\hline Income not revealed & 0.43 & 0 & dummy \\
\hline Age & 43 & 42 & years \\
\hline Sex is female & 0.51 & 1 & dummy \\
\hline Pessimistic beliefs & 0.72 & 1 & dummy \\
\hline Medium level of education ${ }^{c}$ & 0.82 & 1 & dummy \\
\hline High level of education & 0.08 & 0 & dummy \\
\hline
\end{tabular}

${ }^{\text {a}}$ Denotes difference between status quo $(A)$ nd alternatives $\left(B_{j}\right)$, see Section 3.2.

${ }^{\mathrm{b}}$ Difference of squared values, e.g. damage ${ }^{2}=\operatorname{damage}_{B}^{2}-\operatorname{damag}_{A}^{2}$.

${ }^{\mathrm{c}}$ Vocational school, community college, technical college.

and price move in fixed proportions, i.e. if the quantity of power consumed stays constant. Since price elasticities of the household demand for electricity are low in Switzerland (Bonomo et al., 1998), this assumption is justifiable.

Product attributes appear in linear, quadratic, and mixed form. The dummy variables for blackout and waste cannot be squared because of multicollinearity. In keeping with Section 3.2, only the difference in the attributes between scenario B (the alternative scenario) and scenario A (the status quo) are relevant for estimation. Therefore all product attribute variables in Table 2 are differences between $\mathrm{A}$ and $\mathrm{B}$, e.g. damage is defined as damage $_{B}-$ damage $_{A}$ and damage as damage $_{B}^{2}-$ damage $_{A}^{2}$ (note that damage da $^{2} \neq$ $\left.\left(\text { damage }_{B}-\text { damage }_{A}\right)^{2}\right)$.

\subsection{Estimation results}

3.4.1. General findings. The model from Eq. (3) was estimated using a random-effects probit specification. Estimation results for the utility function are displayed in Table 3 as well as in Table 6 of Appendix 5.4.

The linear forms of four out of five product attributes have the expected sign; note that (disposable) income has become a function of outlay and thus constitutes a product attribute. While damage has an unexpected (but insignificant) positive coefficient, the negative sign 
Table 3. Estimation of utility function.

\begin{tabular}{|c|c|c|}
\hline Variables (selection) & Coefficient & S.E. \\
\hline Many blackouts (blackouts) & $-0.26656^{* *}$ & 0.09293 \\
\hline Unresolved waste disposal problems (waste) & $-0.67369^{* *}$ & 0.09702 \\
\hline Damage in 100 CHF bn. (damage) & 0.06612 & 0.15924 \\
\hline Insurance coverage in percent (coverage) & $0.01416^{* *}$ & 0.00329 \\
\hline Disposable income in CHF 000s (income) & $2.9755^{*}$ & 1.4302 \\
\hline Income $^{2}$ & 0.0228 & 0.0142 \\
\hline Damage $^{2}$ & $-0.2320^{* *}$ & 0.0794 \\
\hline Coverage $^{2}$ & $-0.00009^{* *}$ & 0.00003 \\
\hline Damage* coverage & $0.0342^{* *}$ & 0.0106 \\
\hline Coverage*blackouts & $-0.00363^{*}$ & 0.00164 \\
\hline$($ Pessimistic beliefs)* income & -1.0153 & 0.6523 \\
\hline (Medium level education)* income $^{2}$ & $-0.0239^{+}$ & 0.0136 \\
\hline (High level education)* income & $-6.7719^{* *}$ & 1.6609 \\
\hline Age* income & $0.0480^{*}$ & 0.0211 \\
\hline Age $^{*}$ income $^{2}$ & $-0.0002^{+}$ & 0.0001 \\
\hline Constant & $0.22319^{+}$ & 0.13173 \\
\hline \multicolumn{3}{|l|}{ For complete estimation results see Appendix 5.4} \\
\hline Observations & 4119 & \\
\hline Number of individuals & 375 & \\
\hline Log likelihood & -1959.67 & \\
\hline Log likelihood constant only & -2326.77 & \\
\hline$\rho$ & 0.5425 & \\
\hline$\sigma_{\eta}$ & 1.0890 & \\
\hline
\end{tabular}

${ }^{+}$Significant at $10 \%,{ }^{*}$ Significant at $5 \%,{ }^{* *}$ Significant at $1 \%$.

of damage $e^{2}$ changes the overall impact from positive to negative for a large subset of values of explanatory variables.

Most importantly, the coefficients for coverage and income have both a positive and significant effect on utility and hence choice probability. Finally, there is some indication of respondents who hold a more pessimistic belief than experts with regard to the probability of an accident having a lower marginal utility of income over a large range of income (see the not quite significant coefficient of pessimistic beliefs*income). In keeping with Eq. (4) below, this should result in a higher marginal willingness to pay for additional coverage. The same holds for individuals with a high education level, however, the negative basis effect evidenced in Table 3 is so strong that their WTP remains negative over the whole range of coverage.

Finally, the goodness of fit, measured by a pseudo- $R^{2}$ of 0.16 , is satisfactory for a randomeffects specification. The significantly positive value of $\rho=0.54$ shows that 54 percent of the variance of the error term can be attributed to individual-specific effects. 
3.4.2. Calculation of MWP for financial security. The marginal willingness to pay (MWP) for additional coverage is given by

$$
M W P=\frac{\partial \hat{U} / \partial \text { coverage }}{\partial \hat{U} / \text { income }},
$$

with income defined as income net of outlay on electricity so that $\partial \hat{U} / \partial$ income is the estimated marginal utility of disposable income. The ratio between the marginal utility of coverage and the marginal utility of income defines the MWP for additional financial security through increased insurance coverage. In order to express the MWP in terms of US cents per kwh, the quantity of power consumed must be known. This was calculated as the annual outlay devided by the average price of electricity at the household level during the year 1999 (16.2 Swiss cents/kwh, i.e. US 11 cents/kwh), obtaining

$$
\text { MWP[US cents } / \mathrm{kWh}]:=\frac{\text { MWP[US } \$ / \text { year] } \cdot 100}{\text { Outlay in US } \$ / 0.11}
$$

In order to get an impression of the MWP across the entire sample, the MWP for increased coverage was calculated for each person based on his or her specific socioeconomic characteristics and a power type which has few blackouts (blackouts $=0$ ), unresolved problems with waste disposal (waste $=1$ ), a maximum possible loss of CHF $200 \mathrm{bn}$. (US\$ $130 \mathrm{bn}$.) and initial coverage amounting to a mere 1 percent (coverage $=1$ percentage). The resulting density function is shown in Figure 4.

For the calculation of this density function, only the subsample of individuals who stated their income was used. Average MWP for this group amounts to $0.16 \mathrm{US}$ cents/kwh, median

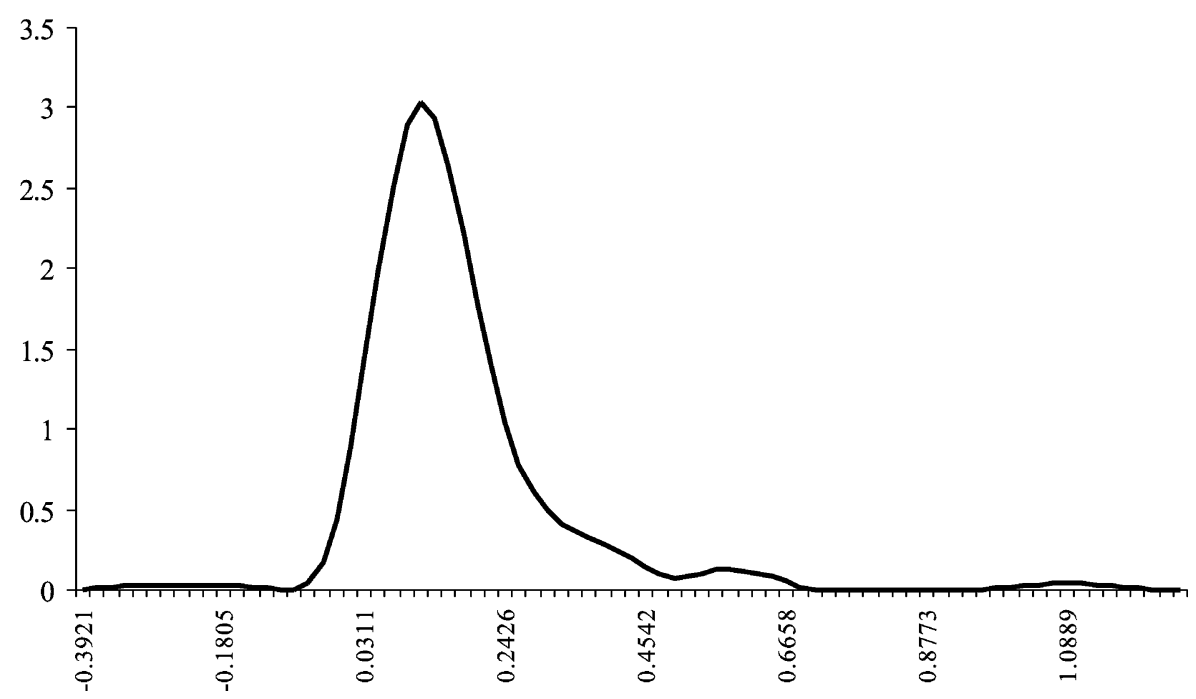

Figure 4. Density of MWP in US cents/kwh. damage $=200$ (US\$ $130 \mathrm{bn}$.), waste $=1$, blackouts $=0$ and coverage $=1$. (Epanechnikov kernel with a bandwidth of 0.66 ). 


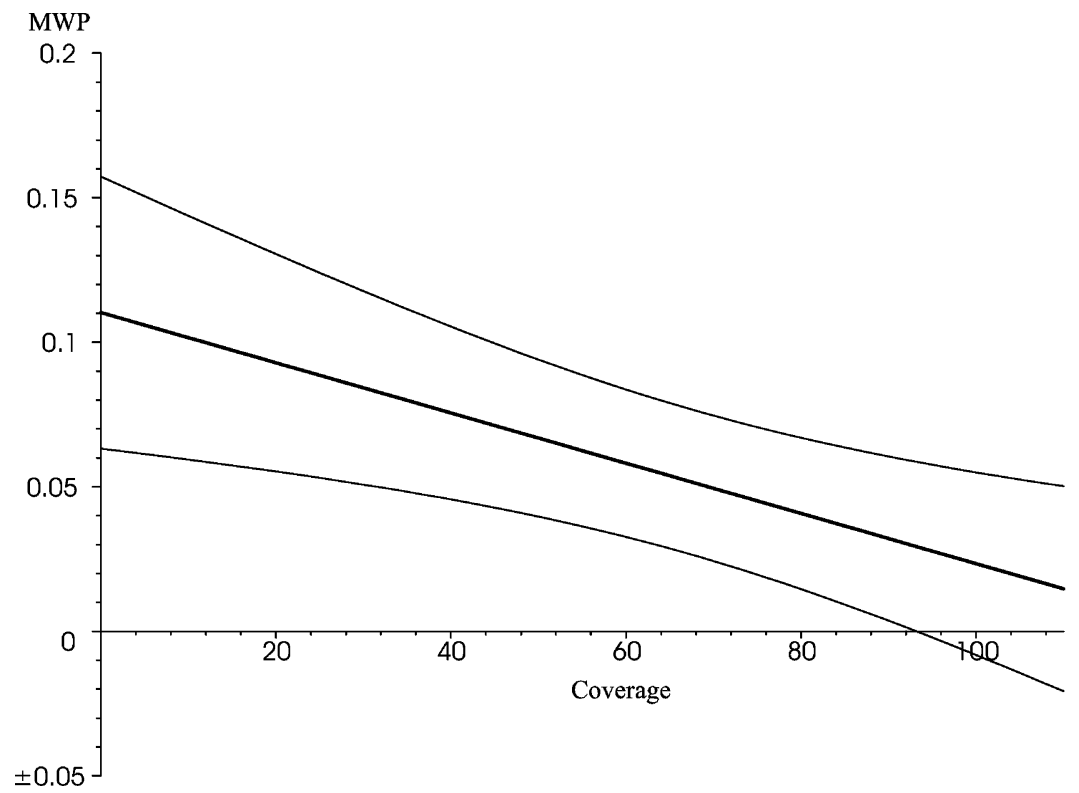

Figure 5. MWP in US cents/kwh including 95 percent confidence intervals; 42 year old woman, medium level education, pessimistic beliefs, income US\$24,000, damage $=200$ (US\$ $130 \mathrm{bn}$.), waste $=1$, blackouts $=0$, outlay $=840($ US\$ 560).

MWP to 0.14 US cents/kwh. Compared to the average price of 11 cents paid in 1999, this corresponds to 1.3 percent of the electricity price. This does not seem excessive a priori, especially when taking the decline of MWP with increasing coverage into account (see Figure 5).

In order to check whether these MWP values are significantly different from zero, standard errors were calculated, using the delta-method although it may result in an underestimate (see e.g. Polsky et al. (1997) and Telser (2002, ch. 4)). The alternatives would have been the Filler method, which only works for a simple ratio of coefficients, and bootstrapping.

Figure 5 shows the MWP of a person with median characteristics (woman aged 44 with medium level of education and pessimistic beliefs) along with its 95 percent confidence intervals. Estimated MWP declines, becoming indistinguishable from zero near an initial coverage rate of 95 percent. At a coverage level of 100 percent at the latest, MWP should theoretically be zero. However, this restriction was in no way built into the experiment. Figure 5 thus may be considered as providing preliminary evidence for the experiment's validity.

3.4.3. Plausibility tests of estimated MWP. A first plausibility test derives from the influence of income on MWP. If financial security is a normal economic good, MWP should be higher than average among individuals with high income. This prediction is borne out in Figure 6. However, these differences lack statistical significance.

Second, due to the income effect, MWP should decline with increasing outlay on electricity. This is indeed the case without exception in both Tables 4 and 5. Moreover, MWP again 


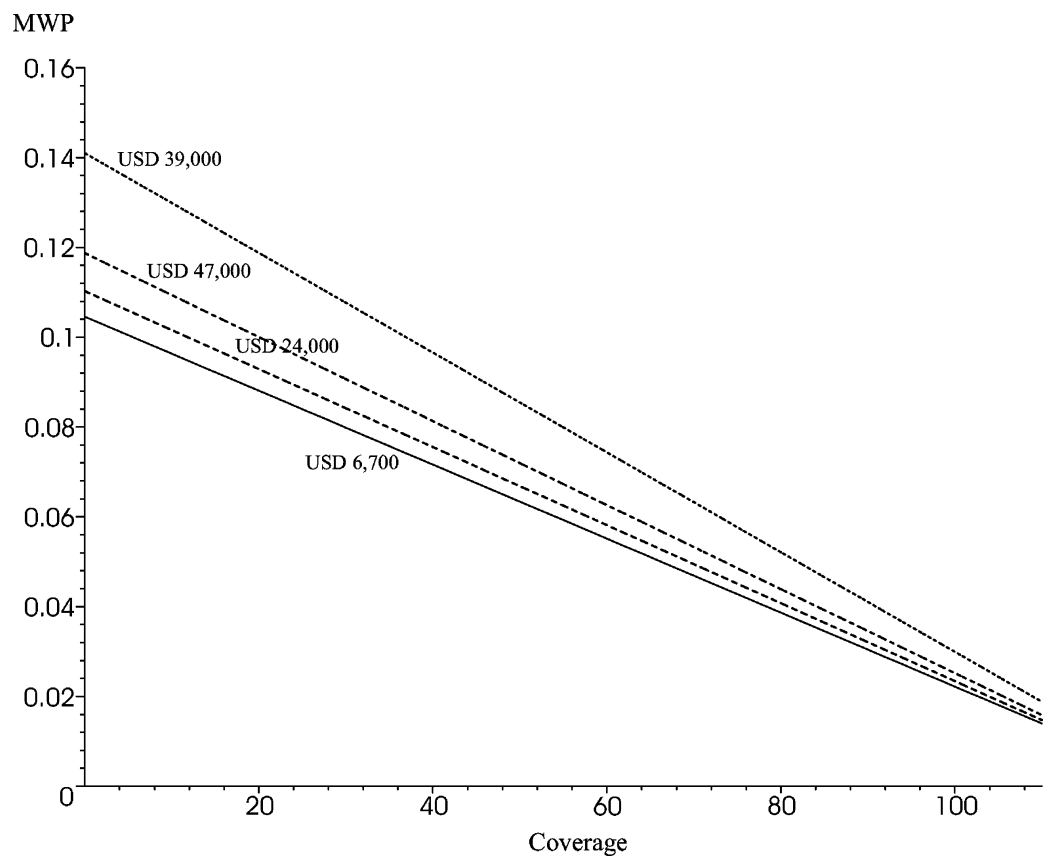

Figure 6. MWP in US cents/kwh of a 42 year old woman with medium level education, pessimistic beliefs and outlay on electricity of CHF 840 (US\$ 560) per year. Maximum loss is CHF 200 bn. (US\$ 130 bn.). Incomes at US\$ 6,700; 24,000; 47,000; 93,000 respectively.

decreases with initial coverage for a given value of electricity outlay, confirming Figures 5 and 6.

Comparison of Tables 4 and 5 (with maximum loss doubled) shows that MWP increases systematically with maximum possible loss. This too corresponds with theoretical considerations if risk aversion is assumed (Chambers and Quiggin, 2000, ch.3).

Finally, under very general conditions, the MWP of a risk averse individual is predicted to increase with increasing probability of an accident. Evaluation of the equation for MWP

Table 4. MWP in US cents/kwh of a 42 years old woman with medium level education, pessimistic beliefs and an income of US\$24,000. Maximum loss is CHF 100 bn. (US\$ 65 bn.), waste $=1$, blackouts $=0$.

\begin{tabular}{cccccccc}
\hline $\begin{array}{l}\text { Coverage } \\
\text { (percent) }\end{array}$ & \multicolumn{2}{c}{ Electricity outlay (US\$ per year) } & & & & \\
\hline 0 & 400 & 530 & 670 & 800 & 930 & 1070 & 1200 \\
\hline 20 & 0.1306 & 0.0979 & 0.0783 & 0.0652 & 0.0559 & 0.0489 & 0.0434 \\
40 & 0.1063 & 0.0797 & 0.0637 & 0.0531 & 0.0455 & 0.0398 & 0.0353 \\
60 & 0.0820 & 0.0615 & 0.0491 & 0.0409 & 0.0351 & 0.0307 & 0.0273 \\
80 & 0.0576 & 0.0432 & 0.0346 & 0.0288 & 0.0247 & 0.0216 & 0.0192 \\
100 & 0.0333 & 0.0250 & 0.0200 & 0.0166 & 0.0142 & 0.0125 & 0.0111 \\
\hline
\end{tabular}


Table 5. MWP in US cents/kwh of a 42 years old woman with medium level of education, pessimistic beliefs and an income of US\$24,000. Maximum loss is CHF $200 \mathrm{bn}$. (US\$ $130 \mathrm{bn}$.), waste $=1$, blackouts $=0$.

\begin{tabular}{cccccccc}
\hline $\begin{array}{l}\text { Coverage } \\
\text { (percent) }\end{array}$ & \multicolumn{2}{c}{ Electricity outlay (US\$ per year) } & & & & \\
& 400 & 530 & 670 & 800 & 930 & 1070 & 1200 \\
\hline 0 & 0.1545 & 0.1158 & 0.0926 & 0.0771 & 0.0661 & 0.0578 & 0.0514 \\
20 & 0.1301 & 0.0976 & 0.0780 & 0.0650 & 0.0557 & 0.0487 & 0.0433 \\
40 & 0.1058 & 0.0793 & 0.0634 & 0.0528 & 0.0453 & 0.0396 & 0.0352 \\
60 & 0.0815 & 0.0611 & 0.0488 & 0.0407 & 0.0349 & 0.0305 & 0.0271 \\
80 & 0.0571 & 0.0428 & 0.0343 & 0.0285 & 0.0244 & 0.0214 & 0.0190 \\
100 & 0.0328 & 0.0246 & 0.0197 & 0.0164 & 0.0140 & 0.0123 & 0.0109 \\
\hline
\end{tabular}

with regard to all relevant levels of the attributes shows that the MWP of individuals with pessimistic beliefs (probability of accident is higher than experts' estimate) is indeed greater than with beliefs congruent with experts' estimate. This effect, however, is not significant.

\subsection{Out-of-sample test}

The two performance criteria of an empirical investigation are its reliability and validity (Singleton and Straits, 1999). Reliability refers to the stability and consistency of the operational definition (here: MWP); validity refers to the goodness of fit between the operational definition and the concept that it is supposed to measure (here: valuation of nuclear risks).

Since effective choices cannot be observed and compared with the stated choices in the experiment, it is not possible to check validity directly, i.e. whether estimated MWP for a reduction of financial risk is a good proxi for the valuation of financial consequences of nuclear risks.

However, there are studies suggesting that the 'stated choice' method leads to results that are in line with corresponding hedonic price estimations (Gegax and Stanley, 1997; Louviere, Meyer, and Bunch, 1999; Haener, Boxall, and Adamowicz, 2000). This suggests validity.

As to reliability, an out-of-sample test can be performed on the 10 percent of observations that were not used for estimation. The model predicts the probability of choosing the alternate scenario. For a calculated probability of more than 50 percent, the individual is assumed to choose the alternate scenario. It turns out that out of sample, roughly 70 percent of all decisions were predicted correctly. This share has to be compared to the share of correct decisions which would result from a random process. In the sample used for estimation, the alternate scenario was chosen 63 percent of the time. Now, a random process that generates choice of the alternate scenario in 63 percent of all cases and of the status quo scenario in 27 percent would predict correctly in 47 percent of cases. This value is the sum of the probability that the random process predicted the alternative and that the alternative was actually chosen $\left(0.63^{2}\right)$ plus the probability that it predicted the status quo and that the status quo was actually chosen $\left(0.27^{2}\right)$. 
The estimated utility model thus serves to increase the share of correct predictions by 23 percentage points $(=70-47)$ over a random process. This points to a measure of reliability.

\section{Conclusions}

Measurement of willingness to pay for an increased internalization of the risks emanating from nuclear power plants is important for energy policy. One instrument of internalization is extending coverage provided by mandatory liability insurance for plant operators. For all its popular appeal, such a proposal will face opposition in parliament and by consumers since higher insurance premiums lead to higher electricity prices.

This study seeks to determine how much Swiss citizens value increased financial security through increased insurance coverage in case of an accident by using the economic concept of marginal willingness to pay for (financial) security.

Since additional coverage is not available to individual consumers, a 'stated choice' experiment was carried out, in which respondents decide in favor of or against an alternative to the status quo, characterized by several attributes of electricity. These attributes are varied throughout the experiment, in contradistinction to conventional 'contingent valuation' approaches. The relevant attributes were established by means of three pretests and turned out to be electricity price, frequency of blackouts, waste disposal, maximum possible loss in case of an accident, and insurance coverage. The econometric analysis confirms this selection, since all attributes are estimated to be statistically significant arguments of the underlying utility function. Average marginal willingness to pay for an additional percentage point of compensation for losses in excess of the status quo amounts to some 0.16 US cents per kwh (median value 0.14 cents), approaching zero when insurance coverage goes towards 100 percent.

Specifically, an increase of mandated liability insurance coverage from today's CHF 0.7 bn. (US\$ $0.47 \mathrm{bn}$.) to CHF $4 \mathrm{bn}$. (US\$ $2.7 \mathrm{bn}$.) would command a WTP amounting to 0.40 US cents/kwh. This can be compared to an estimate of additional cost. In a companion study, a log-logistic density function for nuclear damages (i.e. the loss function for nuclear insurers) was calibrated. According to that study, an increase of liability insurance from today's CHF 0.7 bn. (US\$ 0.47 bn.) to CHF 4 bn. (US\$ 2.7 bn.) would result in an increase in the price of electricity of 0.008 US cents/kwh (Zweifel and Umbricht, 2002, Table 4.16). Therefore, quintuplicating current insurance coverage could lead to a welfare gain for the majority of Swiss citizens.

This proposition has to be qualified in several ways. On the cost side, the choice of the distribution law can be criticized. Indeed, a different choice (Gamma e.g.) would entail somewhat changed marginal cost estimates. On the benefits side investigated here, one has to accept the fact that no thought experiment can simulate the actual decision environment completely. In particular there is no guarantee that participants take described damages seriously and do not speculate on the government providing financial assistance to victims in case of a major accident. ${ }^{2}$ On the other hand, estimated values of marginal willingness to pay do exhibit theoretically plausible variations in several dimensions, thus providing a measure of support for the validity of the experiment. 


\section{Appendix}

\subsection{Contextual information provided for the interview}

\section{Pros and Cons of Nuclear Power}

In the current debate, the following advantages of nuclear power are often mentioned:

- produces a great deal of electric power

- no environmental pollution

- low accident risk compared to other sources of energy

Disadvantages mentioned are:

- degradation of natural scenery

- disposal of nuclear waste

- long-term consequences of accidents (nuclear radiation).

\section{Damages Caused by Accidents in Power Plants}

Power plants can cause great damage in case of an accident. In case of a severe accident in a nuclear power plant, a large part of the resident population needs to be evacuated. Acute disease and death may occur in the vicinity of the power plant. However, only few deaths are expected as a rule. With considerable delay, an accident may affect remote areas in that entire regions suffer from radiation and may partially become uninhabitable for years.

The probability of such an accident is very small.

Breaches of hydro dams are somewhat more frequent. However, their probability is low as well.

A sudden breach of a dam releases a huge flood wave. Affected individuals cannot be warned in time, and thousands of them may die. In most cases however, there is enough time to warn residents, permitting the great majority of them to be evacuated. The immediate risk is limited to people on the downstream side of the dam. Most damages are repaired within a year after the accident. However, the natural environment may take several years to recover. At any rate, damages to man and nature are massive.

Today already, operators of nuclear power plant are mandated to buy insurance that pays in case of damage. However, insurance covers only part of the possible loss. It is an open question as to who would be responsible for the uncovered remainder. Federal parliament would decide on the issue. Therefore, there is no guarantee that victims are fully compensated. The uncovered remainder of the damage may easily exceed Switzerland's annual tax revenue.

In order to better secure compensation of victims the law could stipulate an extension of insurance coverage. In this way a greater part of possible damage would be paid by insurers covering power plants. This would have the advantage of providing improved financial protection to victims. In addition, this could be an incentive for power plant operators to invest even more in the safety of their plant.

On the other hand, an extension of insurance coverage would increase the cost of operation of power plants, resulting in higher prices for electricity to consumers.

Figure 7. Contextual information provided for the interview. 


\subsection{Risk estimate}

Subjective probability of large-scale nuclear accident:

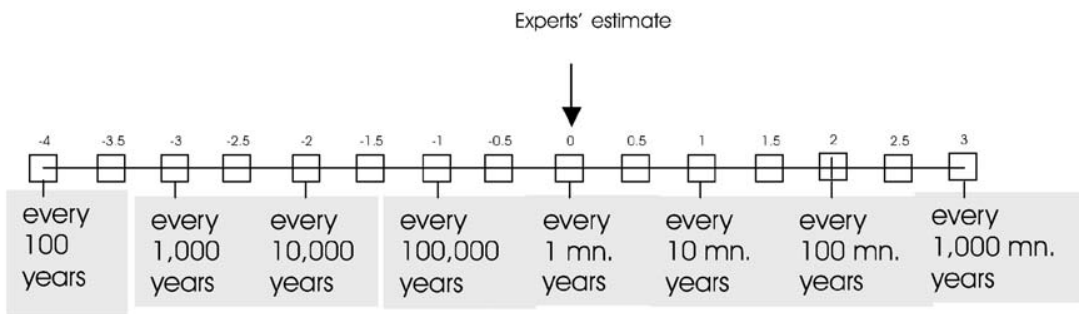

Subjective probability of dam failure (hydro):

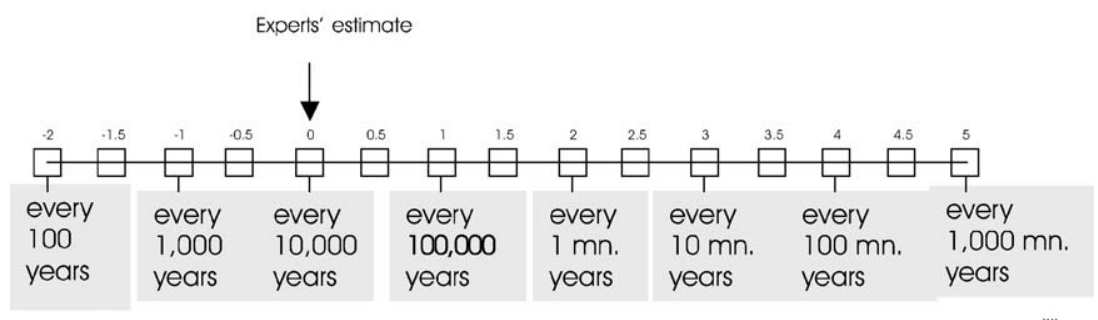

Figure 8. Method used in the questionnaire to elicit the subjective risk estimates for nuclear and hydro accidents respectively. The respondents had to mark their own risk estimate in one of the boxes.

\subsection{Gosset code used to construct reduced design}

The experimental design was constructed using the program 'Gosset' by Hardin and Sloane. For more information on 'Gosset' see Hardin and Sloane (1993) and Hardin and Sloane (1994).

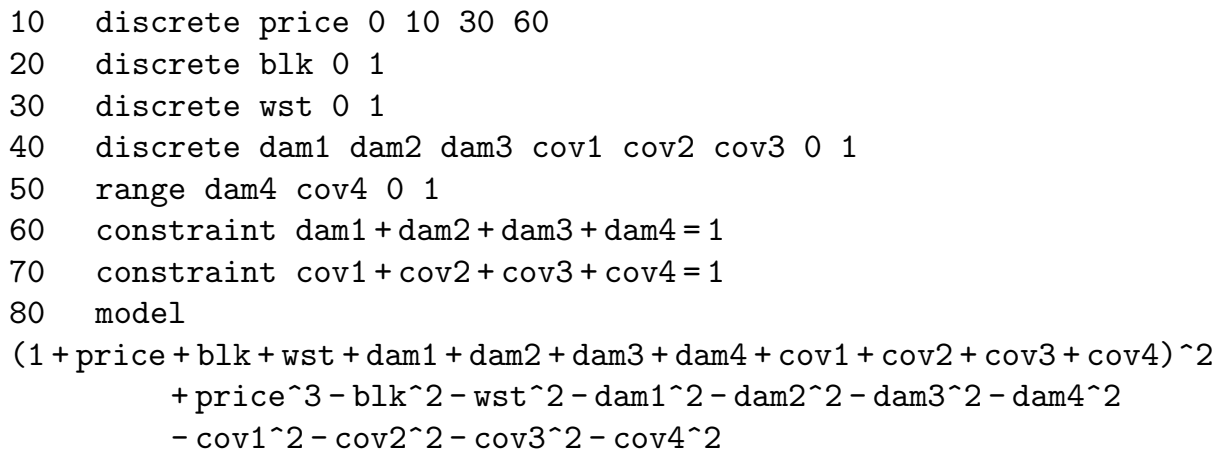




\subsection{Complete estimation results}

Table 6. Complete estimation results. Part 1/2.

\begin{tabular}{|c|c|c|}
\hline Variable & Coefficient & S.E. \\
\hline Many blackouts (blackouts) & $-0.26656^{* *}$ & 0.09293 \\
\hline Unresolved waste disposal problems (waste) & $-0.67369^{* *}$ & 0.09702 \\
\hline Damage in 100 bn. CHF (damage) & 0.06612 & 0.15924 \\
\hline Insurance coverage in percent (coverage) & $0.01416^{* *}$ & 0.00329 \\
\hline Disposable income in CHF 000s (income) & $2.9755^{*}$ & 1.4302 \\
\hline Income $^{2}$ & 0.0228 & 0.0142 \\
\hline Damage $^{2}$ & $-0.2320^{* *}$ & 0.0794 \\
\hline Coverage $^{2}$ & $-0.00009^{* *}$ & 0.00003 \\
\hline Damage $*$ coverage & $0.0342^{* *}$ & 0.0106 \\
\hline Damage $*$ waste & 0.1145 & 0.0771 \\
\hline Damage $*$ blackouts & -0.0364 & 0.0718 \\
\hline Coverage $*$ waste & 0.0012 & 0.0015 \\
\hline Coverage $*$ blackouts & $-0.0036^{*}$ & 0.0016 \\
\hline No income $(=-$ outlay in CHF 000s) & -4.0500 & 2.8300 \\
\hline No income $^{2}\left(=\right.$ outlay $\left.^{2}\right)$ & 0.4740 & 1.0200 \\
\hline (Pessimistic beliefs) $*$ income & -1.0153 & 0.6523 \\
\hline$($ Pessimistic beliefs $) *$ income $^{2}$ & 0.0051 & 0.0031 \\
\hline (Pessimistic beliefs) $*$ noincome & 0.0813 & 0.3305 \\
\hline (Medium level of education) $*$ income & -1.2353 & 1.1717 \\
\hline (Medium level of education) $*$ income $^{2}$ & $-0.0239^{+}$ & 0.0136 \\
\hline (Medium level of education) $*$ noincome & -1.0342 & 2.1487 \\
\hline (Medium level of education) $*$ noincome $^{2}$ & -0.5970 & 0.7770 \\
\hline (High level of education $) *$ income & $-6.7719^{* *}$ & 1.6609 \\
\hline$($ High level of education $) *$ income $^{2}$ & -0.0066 & 0.0146 \\
\hline (High level of education) $*$ noincome & 1.0015 & 2.2493 \\
\hline$($ High level of education $) *$ noincome $^{2}$ & 0.0724 & 0.7700 \\
\hline Age $*$ income & $0.0480^{*}$ & 0.0211 \\
\hline Age $*$ income $^{2}$ & $-0.0002^{+}$ & 0.0001 \\
\hline Age $*$ noincome & -0.0041 & 0.0382 \\
\hline Age $*$ noincome $^{2}$ & 0.00000 & 0.0134 \\
\hline Female $*$ income & 0.0338 & 0.6020 \\
\hline Female $*$ income $^{2}$ & 0.0029 & 0.0032 \\
\hline Female $*$ noincome & 0.4417 & 1.1221 \\
\hline Female $*$ noincome ${ }^{2}$ & -0.4410 & 0.3820 \\
\hline Constant & $0.22319^{+}$ & 0.13173 \\
\hline Observations & 4119 & \\
\hline Number of individuals & 375 & \\
\hline Log likelihood & -1959.67 & \\
\hline Log likelihood constant only & -2326.77 & \\
\hline$\rho$ & 0.5425 & \\
\hline$\sigma_{\eta}$ & 1.0890 & \\
\hline
\end{tabular}

${ }^{+}$significant at $10 \%,{ }^{*}$ significant at $5 \%,{ }^{* *}$ significant at $1 \%$.

Note: Since income is equal to stated income minus outlay on electricity, noincome $=-$ outlay. 


\section{Acknowledgment}

We would like to acknowledge helpful comments and criticism by participants of the Economic Research Seminar of the university of Basel, participants of the 2002 meeting of the Verein für Socialpolitik, as well as two anonymous referees. Financial support came from the Swiss Federal Office of Energy (Berne) and the Swiss Institute of Technology.

\section{Notes}

1. $4 \cdot 2 \cdot 2 \cdot 4 \cdot 4=256$.

2. In fact it is almost certain that the government will step in, as recent much less severe events have shown (e.g. the bailout of Swiss Airlines Ltd. in 2002)

\section{References}

Bonomo, Susanne, Massimo Filippini, and Peter Zweifel. (1998). "Neue Aufschlüsse über die Elektrizitätsnachfrage der schweizerischen Haushalte (New Evidence on Electricity Demand by Swiss Households)," Zeitschrift für Volkswirtschaft und Statistik 134, 415-430.

Camerer, Colin F. and Howard Kunreuther. (1989). "Decision Processes for Low Probability Events: Policy Implications," Journal of Policy Analysis and Management 8, 565-592.

Chambers, Robert G. and John Quiggin. (2000). Uncertainty, Production, Choice and Agency. Cambridge University Press.

Ganderton, Philip T., David S. Brookshire, Michael McKee, Steve Stewart, and Hale Thurston. (2000). "Buying Insurance for Disaster-Type Risks: Experimental Evidence," Journal of Risk and Uncertainty 20, 271289.

Gegax, D. and L. R. Stanley. (1997). "Validating Conjoint and Hedonic Preference Measures: Evidence from Valuing Reductions in Risk," Quarterly Journal of Business and Economics 36, 31-54.

Greene, William H. (1997). Econometric Analysis. Prentice-Hall International.

Haener, M., P. C. Boxall, and W. L. Adamowicz. (2000). "Modeling Recreation Site Choice: Do Hypothetical Choices Reflect Actual Behavior?” Working Paper Staff Paper 00-01, Departement of Rural Economy, University of Alberta, Edmonton, Canada, 49, 1-15.

Hardin, R. H. and N. J. A. Sloane. (1993). “A New Approach to the Construction of Optimal Designs," Journal of Statistical Planning and Inference 37, 339-369.

Hardin, R. H. and N. J. A. Sloane. (1994). "Operating Manual for Gosset: A general purpose program for constructing experimental designs (second edition).” http://www.research.att.com/ njas/gosset/.

Hausman, J. A. (1993). Contingent Valuation-A Critical Assessment. North Holland.

Hedayat, A. S., N. J. A. Sloane, and John Stufken. (1999). Orthogonal Arrays-Theory and Applications. Springer.

Hirschberg, Stephan, G. Spiekerman, and R. Dones. (1998). Severe Accidents in the Energy Sector. Paul Scherrer Insitute, Report 98-16.

Johnson, Reed F. and William H. Desvousges. (1997). "Estimating Stated Preferences with Rated-Pair Data: Environmental, Health, and Employment Effects of Energy Programs," Journal of Environmental Economics and Management 34, 79-99.

Kanninen, Barbara J. (2002). "Optimal Design for Multinomial Choice Experiments," Journal of Marketing Research 39, 214-227.

Krupnick, Alan, Anna Alberini, Maureen Cropper, Nathalie Simon, Bernie O'Brien, Ron Goeree, and Martin Heintzelman. (2002). "Age, Health and the Willingness to Pay for Mortality Risk Reductions: A Contingent Valuation Survey of Ontario Residents," Journal of Risk and Uncertainty 24, 161-186.

Kunreuther, Howard, Nathan Novemsky, and Daniel Kahneman. (2001). "Making Low Probabilities Useful," The Journal of Risk and Uncertainty 23, 103-120. 
Lancaster, Kevin. (1966). “A New Approach to Consumer Theory,” Journal of Political Economy 74, $132-157$.

Louviere, J. J., R. J. Meyer, and D. S. Bunch. (1999). "Combining Sources of Preference Data for Modeling Complex Decision Processes," Marketing Letters 10, 205-217.

Louviere, Jordan J. and David A. Hensher. (1982). "On the Design and Analysis of Simulated or Allocation Experiments in Travel Choice Modelling," Transportation Research Record 890, 11-17.

Louviere, Jordan J., David A. Hensher, and Joffre D. Swait. (2000). Stated Choice Methods-Analysis and Applications. Cambridge University Press.

Magat, Wesley A., W. Kip Viscusi, and Joel Huber. (1988). "Paired Comparison and Contingent Valuation Approaches to Morbidity Risk Valuation," Journal of Environmental Economics and Management 15, 395-411.

McFadden, Daniel. (2001). "Economic Choices," American Economic Review 91, 351-378.

Mitchell, Robert Cameron. (1989). "Using Surveys to Value Public Goods: The Contingent Valuation Method," Ressources for the Future.

Polsky, Daniel, Henry Glick, Richard Willke, and Kevin Schulman. (1997). "Confidence Intervals for CostEffectivness Ratios: A Comparison of Four Methods," Health Economics 6, 243-252.

Shavell, Steven. (1986). "The Judgement Proof Problem," International Review of Law and Economics 6, 45-58.

Singleton, Royce A. Jr. and Bruce C. Straits. (1999). Approaches to Social Research, 3rd edition. Oxford University Press

Telser, Harry. (2002). "Nutzenmessung im Gesundheitswesen-Die Methode der Discrete Choice Experimente (Preference Elicitation in Health Care-The Technique of Discrete Choice Experiments)." University of Zurich, Ph.D. dissertation.

Telser, Harry and Peter Zweifel. (2002). "Measuring Willingness-to-Pay for Risk Reduction: An Application of Conjoint Analysis," Health Economics 11, 129-139.

Viscusi, W. Kip (1998). Rational Risk Policy. Oxford University Press.

Zweifel, Peter and Roland D. Umbricht. (2002). "Verbesserte Deckung des Nuklearrisikos—zu welchen Bedingungen? (Extended Coverage of Nuclear Risk-on What Conditions)," Forschungsprogramm Energiewirtschaftliche Grundlagen, Bundesamt für Energie. 